\title{
Chemical Detection Based on Adsorption-Induced and Photo-Induced Stresses in MEMS Devices
}

\author{
P.G. Datskos ${ }^{a, b}$ \\ a Oak Ridge National Laboratory, P.O. Box 2008. Oak Ridge, TN 37831-8039 \\ ${ }^{b}$ University of Tennessee, 401 Nielsen Physics Building, Knoxville, TN 37996-1200
}

\begin{abstract}
Recently there has been an increasing demand to perform real-time in-situ chemical detection of hazardous materials, contraband chemicals, and explosive chemicals. Currently, real-time chemical detection requires rather large analytical instrumentation that are expensive and complicated to use. The advent of inexpensive mass produced MEMS (micro-electromechanical systems) devices opened-up new possibilities for cthemical detection. For example, microcantilevers were found to respond to chemical stimuli by undergoing changes in their bending and resonance frequency even when a small number of molecules adsorb on their surface. In our present studies, we extended this concept by studying changes in both the adsorptioninduced stress and photo-induced stress as target chemicals adsorb on the surface of microcantilevers. For example, microcantilevers that have adsorbed molecules will undergo photo-induced bending that depends on the number of absorbed molecules on the surface. However, microcantilevers that have undergone photo-induced bending will adsorb molecules on their surfaces in a distinctly different way. Depending on the photon wavelength and microcantilever material, the microcantilever can be made to bend by expanding or contracting the irradiated surface. This is important in cases where the photo-induced stresses can be used to counter any adsorption-induced stresses and increase the dynamic range. Coating the surface of the microstructure with a different material can provide chemical specificity for the target chemicals. However, by selecting appropriate photon wavelengths we can change the chemical selectivity due to the introduction of new surface states in the MEMS device. We will present and discuss our results on the use of adsorption-indueed and photo-induced bending of microcantilevers for chemical detection.
\end{abstract}

Keywords: MEMS, photo-induced stress, electron-hole pairs, adsorption-induced stress, resonance frequency, bimaterial bending

\section{INTRODUCTION}

Present day chemical detection technologies have been based primarily on adaptation of laboratory instruments. For example, a large number of spectroscopic methods have been introduced over the past twenty years that are based upon optical absorption, laser scattering, luminescence, atomic fluorescence spectroscopy or refractive index changes. However, these methods rely on performing laboratory chemical analyses on extracted samples and provide no real-time data for operational feedback, are generally expensive and complicated to use.

Recently there has been an increasing demand to perform real-time in-situ chemical detection of hazardous materials, contraband chemicals, and explosive chemicals, with a simple, effective and easy to use chemical sensor. Gravimetric based chemical sensors achieve specificity with the use of chemically selective coatings. Selective coatings have been used in many different types of gravimetric sensors to sorb various gaseous analytes. Microcantilever-based chemical sensors were found $t$ o respond to chemical stimuli by undergoing changes in their bending and resonance frequency even when a small number of molecules adsorb on their surface. When molecules adsorb on a microcantilever surface, they introduce a differential surface

Further author information -

P.G.D. (Correspondence): E-mail: datskos@utk.edu; Telephone: (423) 574-6205; Fax: (423) 574-9407 
stress. The response of a microcantilever to chemical stimuli is depicted schematically in Figure 1 . Surface stresses $s_{1}$ and $s_{2}$ are balanced at equilibrium, generating a radial force $F_{r}$ along the medial plane of the microcantilever. These stresses become unequal upon exposure to chemicals, producing a bending force, $F_{z}$ that displaces the tip of the microcantitever. This method of chemical detection requires accurate measurements in positional changes of the microcantilever. Previous work has shown that microcantilever bending can be determined with high sensitivity by a number of methods, including optical, capacitive, piezoresistive, and electron tunneling [1]. The metal-coated microcantilevers that are commonly employed in atomic force microscopy (AFM) allow sub-Angstrom $\left(<10^{-10}\right.$ meter) sensitivity to be routinely obtained. For example, Hansma [2] and Binnig [3] have demonstrated AFM sensitivities of $10^{-11} \mathrm{~N}$, corresponding to bending magnitudes of approximately $5 \times 10^{-11} \mathrm{~m}$. More recently, even smaller microcantilever deflections were measured with a resolution of $0.4 \times 10^{-12} \mathrm{~m}[4]$. Standard AFM microcantilevers are typically $100-200 \mu \mathrm{m}$ long. $0.3-3 \mu \mathrm{m}$ thick and 10-30 $\mu \mathrm{m}$ wide, and can be fabricated from various dielectric or semiconducting materials. Microcantilevers made out of GaAs were also fabricated with a thickness of merely 100 nm [5].

In this work, we extended the initial microcantilever based chemical detection by studying changes in both the adsorptioninduced stress and photo-induced stress as target chemicals adsorb on the surface of microcantilevers. We used a novel method to detect and monitor stress changes in bimateria] microcantilevers due to the adsorption of molecules. Microcantilevers that have adsorbed molecules will undergo photo-induced bending that depends on the number of absorbed molecules on the surface. However, microcantilevers that have undergone photo-induced bending will adsorb differently moleccules on their surfaces mainly due to the preexisting surface stress generated by the photons. Depending on the photon wavelength and microcantilever material, the microcantilever can be made to bend by expanding or contracting the irradiated surface. This is important in cases where the photo-induced stresses can be used to counter any adsorption-induced stresses and increase the dynamic range. Although coating the surface of the microstructure with different materials can provide chemical specificity, by choosing appropriate photon wavelengths we can alter the chemical selectivity due to differentiated photoinduced response.

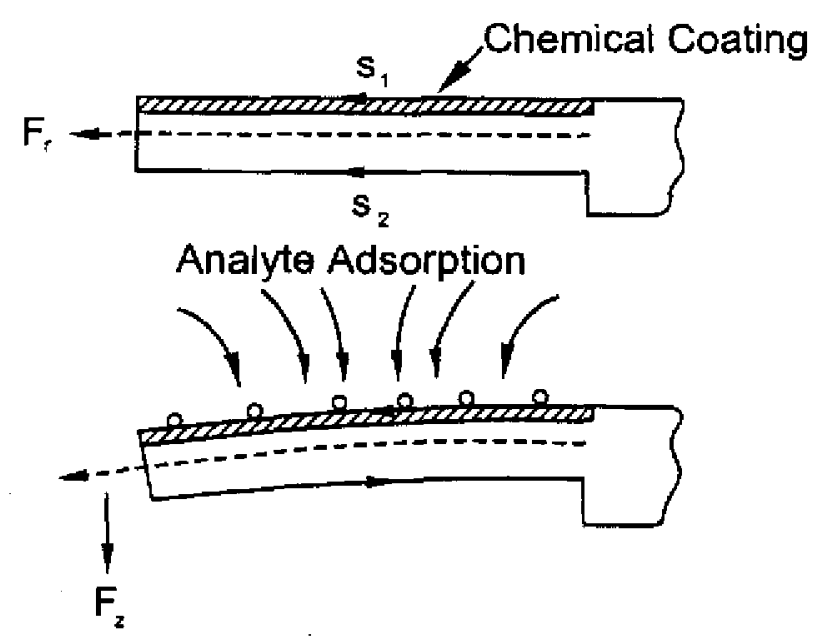

Figure 1. Schematic diagram depicting the bending response of a microcantilever to chemical adsorption. Surface stresses $s_{1}$ and $s_{2}$ are balanced at equilibrium but become unequal and cause bending upon adsorption of analyte molecules. A force along the z-direction is induced due to the differential surface stress, which causes the microcantilever to change its radius of curvature.

In the present studies, we exposed gold-coated microcantilevers to thiol compounds that led to the formation of selfassembled monolyers. This mechanism itself can serve as a very sensitive chemical detection means. However, exposure of microcantilevers with self-assembled monolyers to copper atoms results in the formation of a sensitive chemical layer that can transiently adsorb organophosphorous compounds such as chemical warfare agents.

\section{MOLECULAR ADSORPTION ON MICROSURFACES}

\subsection{A New Method for Chemical Detection}

We have developed a new method for chemical detection based on the photo-induced stress in semiconductor microstructures exposed to chemicals. It is based on the fact that the photo-induced stress in a microstructure depends on the amount of chemical analyte adsorbed on a surface. This method can be used to measure the bending of microcantilevers in an AC mode by modulating the excitation photon beam. It has been shown that microcantilevers will undergo bending following the absorption of photons $[6,7]$. The photo-induced bending is caused by the differential surface stress developed between the top (side exposed to photons) and the bottom (unexposed side) of the microcantilever. For the same number of photons absorbed the photo-induced bending will differ for microcantilevers that have adsorbed different amount of chemicals on their surfaces. This is a consequence of the fact that adsorbed molecules introduce surface stresses themselves. Therefore, microcantilevers that have adsorbed molecules on their surfaces will undergo photo-induced bending that depends on the amount of analyte 


$$
\frac{l}{R}=\frac{2(1-v)}{E t} \frac{d \varepsilon_{B}}{d P} \Delta n+\frac{6(1-v)}{t} a \Delta T
$$

present on their surface. One very attractive feature of the photo-induced bending method is the fact that depending on the photon wavelength used, the direction of the bending can be controlled. For example, when Si microcantilevers are irradiated with photons below the bandgap (for example $\lambda=1300 \mathrm{~nm}$ ), Si heats up resulting in an expansion of the microcantilever and it bends in one direction. However, when photons with energies above the bandgap are used (for example $\lambda=790 \mathrm{~nm}$ ), $\mathrm{Si}$ contracts and the coated microcantilever bends in the opposite direction. This is very important especially in cases where the dynamic range needs to be increased.

When the photo-induced bending is used as the detection method, the chemically selective coating does not have to be restricted to only one surface, as is traditionally the case with gravimetric chemical sensors such as microcantilever [8-11]. Coating both sides with a chemically sensitive layer will essentially increase (double) the effective area available for adsorption of analytes. Since these microstructures are also very sensitive temperature sensors, care should be taken to account for bending due to heat dissipation. One approach would be to employ reference cantilevers that are not exposed to analytes and perform differential measurements.

\subsection{Microcantilever Bending due to Electronically-Induced Stress}

Microcantilevers (see Figure 2) can undergo bending due to the differential surface stress $\left(\Delta s=s_{1}-s_{2}\right)$ created by transient expansions; the top (photon exposed) side expands sooner than the bottom (unexposed) side thus creating a differential surface stress. Earlier work has shown that the absorption of photons by a solid results in temperature changes and thermal expansion which in turn gives rise to acoustic waves at frequencies corresponding to the amplitude modulation of the incident photon beam [12]. It has been shown that in a semiconductor the generation of "free" charge carriers (electrons and holes) via photon irradiation results in the development of a local mechanical strain [12]. This additional strain adds to other strains resulting from temperature changes of the semiconductor. In a semiconductor structure of
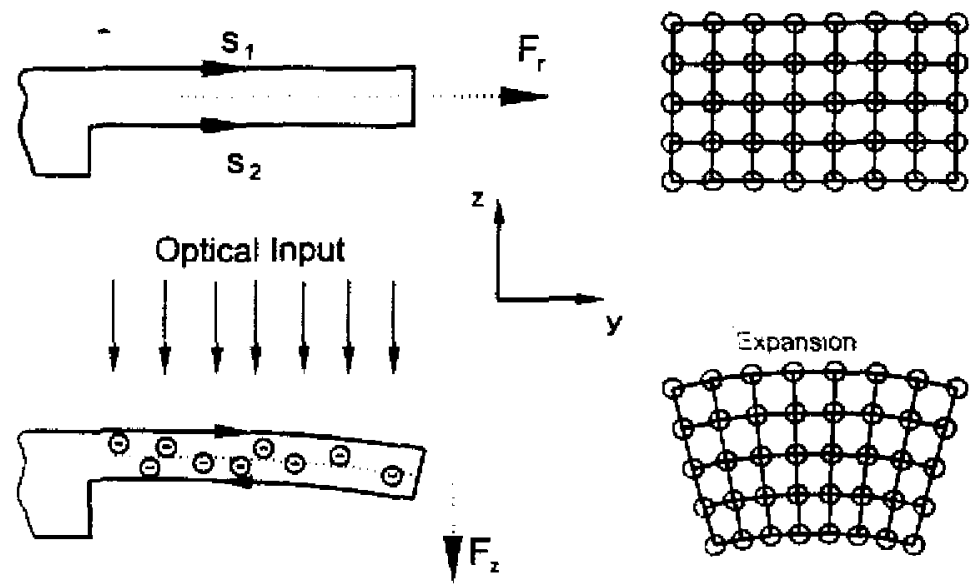

Figure 2. Schematic diagram showing the bending process of a semiconductor microcantilever exposed to photons. Surface stresses $s_{1}$ and $s_{2}$ are balanced at equilibrium. Also depicted is the accompanied expansion of the semiconductor lattice following the generation of electron pairs.

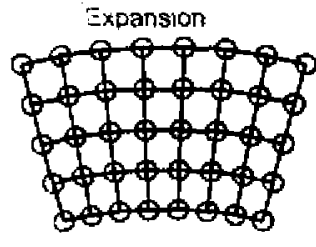

$$
\Delta s=\Delta_{s_{p i}}+\Delta s_{\text {sh }}=\left(\frac{1}{3} \frac{d \varepsilon_{8}}{d P} \Delta n\right) E+\alpha \Delta T E
$$

thickness $t$ and energy bandgap $\varepsilon_{g}$, the change in total surface stress, $\Delta s$, due to photogenerated excess charge carriers, $\Delta n$, and changes in temperature, $\Delta T$, will be the sum of the additional photo-induced stress, $\Delta s_{p i}$, and thermal stress, $\Delta s_{i h}$, viz. [12]

where, $d \varepsilon_{x} / d P$ is the pressure dependence of the energy bandgap, $\alpha$ is the coefficient of themal expansion, and $E$ is the Young's modulus. A hole (in the valence band) decreases the energy of covalent bonds while an electron adds to the bonding (or antibonding) energy. Therefore there is a competing effect between the thermal and the photo-induced stress. When $\mathrm{d} \varepsilon_{g} / \mathrm{d} P$ is negative the photo-induced stress is of opposite sign than that of the thermal stress and will tend to make the semiconductor crystal contract. For a rectangular bar (Figure 2) of length $l$, width $w$, and thickness $t$, the reciprocal of the radius of curvature, $R$, is given by Stoney's relationship [12-15] 
where, $v$ is the Poisson's ratio. The reciprocal of the radius of curvature is approximately equal to $d^{2} z / d y^{2}$. Then the maximum displacement $z_{\max }$ of the microcantilever is given by

$$
=\frac{(1-v) l^{2}}{t} \frac{d \varepsilon_{g}}{d P} \Delta n+\frac{3(1-v) l^{2}}{t} \alpha \Delta T
$$

The first term inside of Eqn (3) is due to photo-induced surface stress and the second term is due to thermal stress caused by temperature changes. Neglecting any temperature effects that are only important in relative long time scales (comparable to the thermal time constant $>\mathrm{ms}$ ), $z_{\max }$ can be written as

$$
z_{\max }=\frac{(l-t) l^{2}}{t} \frac{d \varepsilon_{g}}{d P} \Delta n
$$

Assuming that an incident radiant power, $\Phi_{e}$, on a semiconducter microcantilever generates a number density of excess charge carriers, $\Delta n$, we get

$$
\Delta n=\eta \frac{\lambda}{h c l w l} \frac{\tau_{L}}{l w} \Phi_{e}^{a b s}
$$

where $\eta$ is the quantum efficiency, $h\left(=6.625 \times 10^{-34} \mathrm{~J} \mathrm{~s}\right)$ is Planck's constant, $c\left(=3 \times 10^{8} \mathrm{~m} \mathrm{~s}^{-1}\right)$ is the speed of light, and $\tau_{L}$ is the lifetime of the carriers in the semiconductor. Then the maximum displacement $z_{\max }$ can be rewritten as

$$
z_{\max }=\frac{(1-\nu) t^{2}}{t}\left(\eta \frac{\lambda}{h c} \frac{d \varepsilon_{g}}{d P} \frac{1}{l w t}+\frac{3 \alpha}{m c_{p}} \tau_{L} \Phi_{e}\right.
$$

We can define the deflection responsivity $\Re=z / \Phi_{e}$ viz.,

$$
\Re=\frac{(1-v) l}{w t^{2}} \frac{\lambda}{h c} \frac{d \varepsilon_{g}}{d P} \eta \tau_{L}
$$

Since the charge carriers can be generated in a very short time the photo-induced stress can manifest itself much faster than thermal stress. Of course, the overall change in $z_{\max }$. will depend on the physical and mechanical properties of the semiconductor. In fact, for the same input power a Si microcantilever exhibits a photo-induced bending that is about 4 times larger than that due to thermally-induced stress. When applied to the problem of chemical sensing, this approach provides a fast an efficient method to detect the presence of target molecules as they adsorb on a microcantilever surface. 


\subsection{Chemical Sensing Based on Microcantilever Resonance Frequency Shifts}

Currently available gravimetric chemical sensors $[16,17]$, such as quartz crystal microbalances, surface acoustic wave (SAW), acoustic plate mode (APM) devices; chemiresitors, and flexural plate wave oscillators (FPW), achieve sensing by monitoring the sorption processes on the sensing element that results in some frequency shift. When the interaction of an analyte with the microcantilever results in the adsorption of that species on the microcantilever surface, it causes changes in the deflection (or radius of curvature) and the resonant frequency, $f_{0}$, of the microcantilever. For a rectangular microcantilever bar with a spring constant, $k$, the resonance frequency, $f_{0}$, is inversely proportional to the square root of the effective mass, $m_{e f f}$, of the microcantilever $\left[f_{0}=(1 / 2 \pi)\left(k / m_{e f l}\right)^{1 / 2}\right]$. The effective mass can be related to the mass of the microcantilever beam, $m_{b}$, through the relation: $m_{e f f}=n_{\theta} m_{t}$ where $n_{0}$ is a geometrical factor $[1,18]$. For example, in the case of commercially available silicon nitride $\left(\mathrm{SiN}_{\mathrm{x}}\right)$ microcantilevers with spring constants of 0.06 and $0.03 \mathrm{~N} / \mathrm{m}$, the values of $n_{\theta}$ are 0.14 and 0.18 respectively [18]. In order to determine the change in resonance frequency, $\Delta f\left(m_{e f f}, k\right)$, both the change in spring constant, $\Delta k$, and mass, $\Delta m_{e f f}$ must be obtained; a change of $\Delta k / k \approx 10^{-6}$ was reported for gelatin coated microcantilevers [8]. For a rectangular microcantilever of length $l$, thickness $t$ and width $w$ the spring constant can be written as [1]

$$
k=\frac{E w t^{3}}{4 I^{3}}
$$

where $E$ is the Young's modulus. When there is a uniform mass load (either from vapors or solution) distributed over the surface, the resonance frequency decreases with increasing mass and can be described by [1]

$$
\frac{d f}{d m}=\frac{1}{4 \pi n_{1} l^{3} w} \sqrt{\frac{E}{\rho^{3}}}
$$

where $\rho[=m /(l w b)]$ is the mass density of the microcantilever and the deposited mass and $n_{1}$ is a geometrical factor approximately equal to unity for this case [1]. For a typical $\operatorname{SiN}_{\mathrm{x}}$ microcantilever (with $E=3.85 \times 10^{12} \mathrm{dyn}_{\mathrm{cm}} \mathrm{cm}^{2}, l=200 \mu \mathrm{m}, w$ $=20 \mu \mathrm{m}, t=0.6 \mu \mathrm{m}, p=3.1 \mathrm{~g} / \mathrm{cm}^{3}$, and $n_{1}=0.98$ ) the expected rate of frequency change will be $d f d m=1.82 \times 10^{12} \mathrm{~Hz} / \mathrm{g}$. Thus, a shift in the resonance frequency will be observed as additional mass is deposited on the microcantilever. Using shorter and nanower microcantilevers can lead to a more sensitive response.

\subsection{Chemical Sensing Based on Microcantilever Bending}

The bending of a microcantilever is extremely sensitive to the adsorption of chemical analytes on its surface, and our results show that monitoring the bending as a function of analytes adsorption provides the highest chemical sensitivity. The minimum detectable mass can be two orders of magnitude smaller when the microcantilever bending is used as the chemical sensing mechanism instead of the resonance frequency change. As microcantilevers are stressed (for example, due to adsorption of a chemical anaiyte) a change in the radius of curvature (see Figure 1) occurs which is given by [13-15]

$$
\frac{1}{R}=\frac{6(1-v)}{E t^{2}} \Delta s
$$

where $R$ is the radius of curvature of the microcantilever, $v$ is the Poisson's ratio and $\Delta s\left(=\Delta s_{1}-\Delta s_{2}\right)$ is the surface differential stress. The surface differential stress $\Delta s$ is proportional (at least to a first order approximation) to the number of adsorbed molecules and therefore to the total additional mass $\Delta m_{a}$, i.e. .

$$
\Delta s=C_{1} \Delta m_{a}
$$


where $C_{1}$ is a proportionality constant that depends on the sticking coefficient of the adsorbed molecules; a typical value for $C_{l}$ is $-10^{15}$ dyn $\mathrm{cm}^{-1} \mathrm{~g}^{-1}$. The bending $z_{\text {max }}\left[=l^{2} /(2 R)\right]$, is related to the additional mass (and differential surface stress) through

Therefore, measurement of the bending $z_{\max }$ allows the amount of adsorbed mass to be determined when the adsorption process is confined to only one side of the microcantilever.

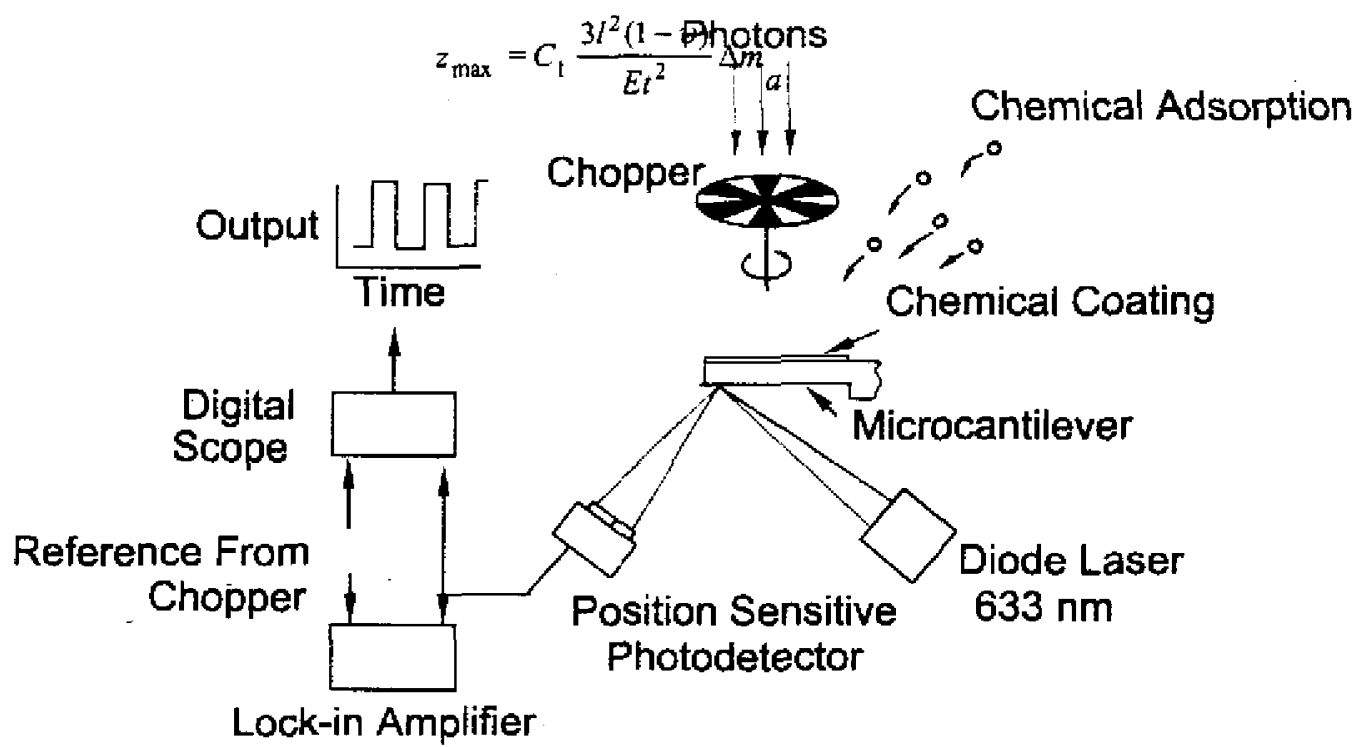

Figure 3. Schemitic of the setup that will be used to determine the photo-induced bending of microcantilevers exposed to analytes. In this arrangement the diode laser with $\lambda=790 \mathrm{~nm}$ (or $1400 \mathrm{~nm}$ ) is used to produce the photo-induced bending and the diode laser with $\lambda=633 \mathrm{~nm}$ is used to measure the deflection of the microcantilever

\section{EXPERIMENTAL}

Although the bending of microcantilevers can readily be determined by a number of means (optical, capacitive, electron tunneling, and piezoresistive) in this work, we employed optical readout techniques. The approach used was adapted from standard atomic force microscopy (AFM) imaging systems, and is shown schematically in Figure 3. A diode laser (delivering $1 \mathrm{~mW}$ at $670 \mathrm{~nm}$ ) was used in a probe configuration to monitor the microcantilever bending and was focused onto the tip of the microcantilever. A quad-element (A,B,C,D) photodiode detector was used to collect the reflected probe beam. The current output ( $i_{A, B C D}$ ) of the photodiode depended linearly on the bending of the microcantilever. A high narrow bandpass optical filter was placed in front of the photodiode allowing the laser beam to be detected while preventing other wavelengths from reaching the photodiode. The amplified differential current signal from the quad cell photodiode, $i_{A, B, C D}\left[=\left(i_{A}+i_{B}\right)-\left(i_{C}+i_{D}\right) /\left(i_{A}+i_{B}+\right.\right.$ $\left.i_{C}+i_{D}\right)$, was monitored and recorded using a digital oscilloscope (TDS 780, Tektronix) or sent to a lock-in amplifier (SR850, Stanford Research Systems) for signal extraction and averaging. A 16 bit analog-to-digital (A/D) converter with a resolution of $0.33 \mathrm{mV}$ was used to monitor the mictocantilever deflection signal and the sampling of the deflection signal was performed at a rate of $4 \mathrm{~Hz}$. A second laser (HeNe, delivering $3 \mathrm{~mW}$ at $633 \mathrm{~nm}$ ) was used to produce the photo-induced stress in the microcantilever. From the measured photodiode signal $i_{\text {A.B.c. }}$, the microcantilever deflection, $z_{\max }$, can be obtained from

$$
z_{\max } \approx \frac{d}{h} l
$$


where $h$ is the distance between the microcantilever and the photodiode, and $d$ is the distance the reflected beam moved on the photodiode (see Figure 4). Taking into account that $d=D / 2 i_{A, B, C, D}$ where $D$ is the diameter of the photodiode, the microcantilever deflection $z_{\max }$ can be determined from

In this work, gold-coated microcantilevers surfaces were derivitized by forming self-assembled composite nonolayers. We did this by placing the microcantilevers in a $1 \mathrm{mM}$ solution of $\$ 1$-mercaptoundecanoic acid/hydrogen peroxide for about 8-12 hours. Following the rinsing of the surfaces with ethanol and water, the microcantilevers were submerged in a $2 \mathrm{mM}$ solution of copper (II) perchlorate hexahydrate / ethanol for about 5-10 minutes. This process resulted in the formation of a

$$
z_{\max }=\frac{1}{2} \frac{\left(i_{A}+i_{B}\right)-\left(i_{C}+i_{d}\right)_{D}}{i_{A}+i_{B}+i_{C}+i_{D}} \frac{{ }_{i}}{h}
$$

carboxylate-terminated n-alkanethiol monolayer on the surface of the microcantilever and bonded copper (II) ions to the surface of the self-assembled monolayer. The copper (II) ions allowed for selective and reversible binding of organophosphonates such as diisopropyl methylphosphonate (DIMP).

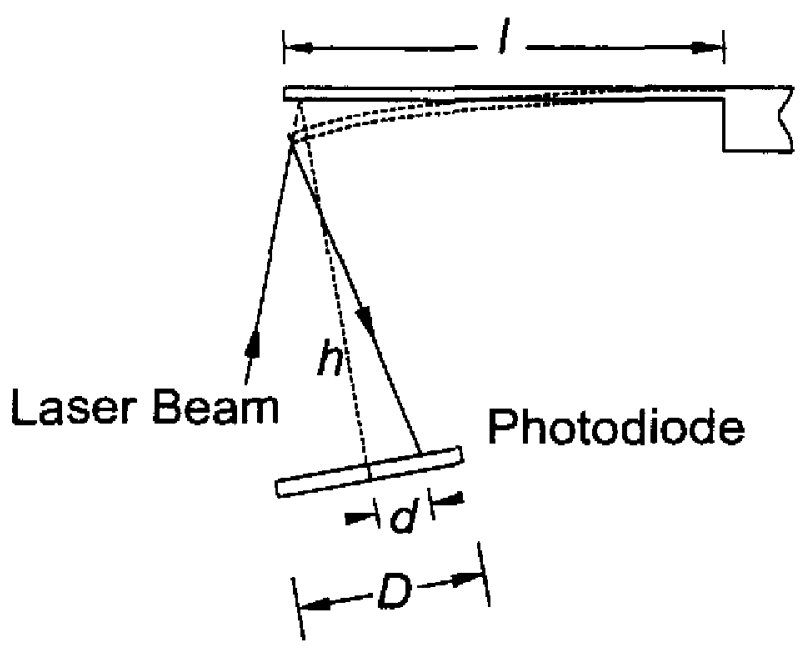

Figure 4. Geometry of the microcantilever bending.

\subsection{2-Mercaptoehtanol}

\section{RESULTS AND DISCUSSION}

Mercaptans interact strongly with gold surfaces and form self-assembled monolayers. The microcantilever deflection, $z_{\max }$, is proportional to adsorption-induced surface stresses which, in turn, is proportional to the number of adsorbed molecules. Therefore by monitoring the microcantilever deflection as a function of time allows the determination of the molecular adsorption rate. In order to demonstrate that microcantilever-based chemical detection can poses a rather large dynamic range, we exposed gold-coated $\mathrm{SiN}_{\mathrm{x}}$ microcantilevers [19] to a mixture of $4.2 \mathrm{mTorr}$ of 2 -mercaptoethanol in $760 \mathrm{Torr}$ of ambient air. In Figure 5 we plotted the deflection as a function of exposure time. This is a rather high concentration of 2-mercaptoethano] and corresponds to 5.5 parts per million ( $(\mathrm{ppm})$. The time $t_{0}=0$ corresponds to the admittance time of 2 -mercaptoethanol into the chamber. The microcantilever reached a bending of $-2606 \mathrm{~nm}$ for an exposure time of $t_{1}=5744 \mathrm{~s}$. At $t=t_{1}$ the chamber containing the microcantilever was flushed with $\mathrm{N}_{2}$. No further bending was observed after equilibrium was reached. As seen from Figure 5, the deflection rate stops as the 2-mercaptoethanol is removed from the sampling volume. The data presented 
in Figure 5 demonstrate both the high sensitivity and large dynamic range of deflection when gold-coated microcantilevers are exposed to 2 -mercaptoethanol vapor.

Adsorption-induced surface stress can be used as a sensitive way to determine the chemical presence of molecular species. In Figure 6 we plotted the time evolution of the microcantilever deflection when exposed to varying small amounts of 2 -mercaptoethanol ranging from 65 to $350 \mathrm{ppb}$. The time $t=0$ corresponds to the time 2-mercaptoethanol was introduced into the chamber. It can be seen that after the introduction of the 2mercaptoethanol into the chamber, a rapid (and appreciable) response of the microcantilever was observed. After final equilibrium was reached, the deflection reached a maximum value that remained constant for each concentration. The mixture in the chamber was sampled and introduced into a Finnigan Mat GCQ gas chromatograph/mass analyzer to determine the amount of 2 mercaptoethanol introduced into the exposure chamber [19]. Figure 7 shows the final deflection of the microcantilever as afunction of the concentration of 2-mercaptoethanol for each exposure. The measured deflection of the microcantilever exhibits a linear dependence on the concentration of 2-mercaptoethanol with a slope of $0.432 \mathrm{~nm} / \mathrm{pp} b$; concentrations as small as $65 \mathrm{ppb}$ can easily be detected using a microcantilever sensor. It can be seen that the microcantilever responds very sensitively to the presence of minute amounts of 2-mercaptoethanol as these molecules adsorb on the gold-coated surface forming self-assembled monolayers.

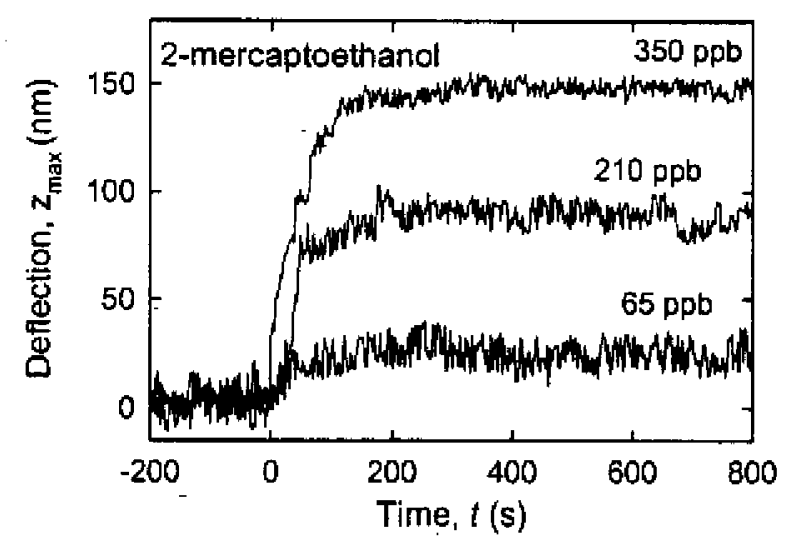

Figure 7. Microcantilever bending as a function of exposure time to 2-mercaptoethanol vapors corresponding to 65,210 , and $350 p p b$; the time $t=0$ corresponds to the time 2-mercaptoethanol was introduced into the detection system.

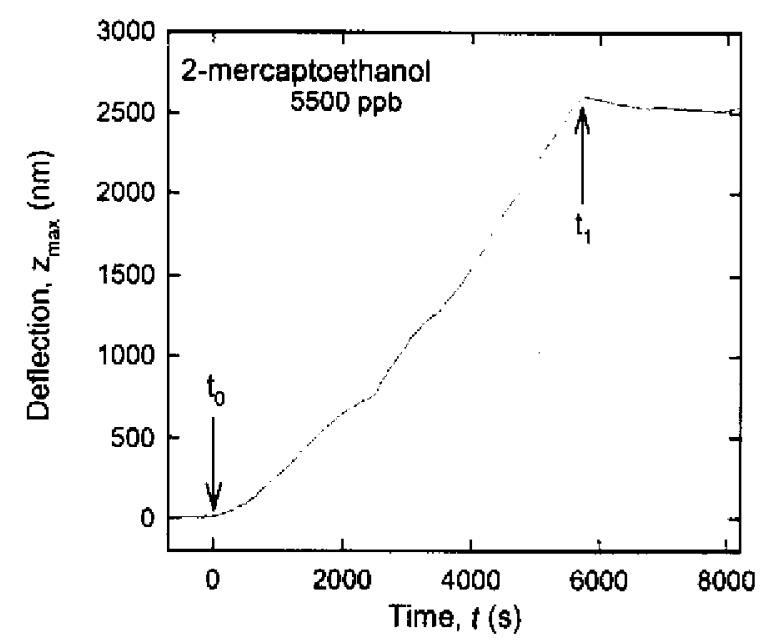

Figure 6. Microcantilever bending as a function of exposure time to 2-mercaptoethanol vapors corresponding to $4.2 \mathrm{mTorr}$ of 2-mercaptoethanol in 760 Tor of ambient air. The time $t=$ $t_{1}$ corresponds to the time the chamber was flushed with $\mathrm{N}_{2}$. 


\subsection{Diisopropyl methylphosphonate (DIMP)}

In our studies we used gold-coated Si microcantilevers to investigate the effect of adsorption of DIMP on the photoinduced bending of microcantilevers. Gold coated surfaces do adsorb DIMP very effectively, but the selectivity can be substantially improved by first coating the surface with selfassembled monolayers which were found were found to adsorb DIMP very effectively [20]. The chemical coating approach used in the present work follows Kepley et al. [20] and is based on self-assembled monolayers. The chemical selective coating capitalizes on the interaction between organophosphonate compounds and a self-assembled based monolayer. The chemical selectivity of the layer is based on the interaction of $\mathrm{Cu}^{+2}$ bound to the microcantilever surface by a carboxylateterminated n-alkanethiol monolayer. Kepley et al. used this kind of chemical coating on a QCM device and based their choice on the fact that $\mathrm{Cu}^{+2}$ and some of its chelates are hydrolysis catalysts for certain chemical warfare agents [20]. Therefore, a chemical surface layer of coordinatively unsaturated $\mathrm{Cu}^{+2}$ can. provide both selective and reversible binding sites for organophosphonate compounds. After a surface cleaning procedure, the microcantilevers wete placed in the $1 \mathrm{mM} 11$ mercaptoundecanoic acid / ethanol solution for a 24 hour period and were then placed in the $2 \mathrm{mM} \mathrm{Cu}(\mathrm{ClO} 4)_{2} * 6 \mathrm{H} 2 \mathrm{O} /$ ethanol solution for about 15 minutes. The time allowed for the composite monolayer to form was not excessive because it is selfassembled. The self-assembly process ceases when the coating layer is one molecule thick and produces a highly ordered monolayer. Microcantilevers with such surface coatings were exposed DIMP molecules by flowing a mixture of $\mathrm{N}_{2}$ and DIMP vapor in a chamber containing the microcantilever. The composite self-assembled monolayer coating transiently adsorbs molecules of DIMP vapor, which causes the microcantilever to bend. We found that our derivitized microcantilevers respond proportionally and reversibly to the presence of DIMP molecules in a way that is distinguishable from any response to common organic solvents such as ethanol, methanol, or acetone. In Figure 8 we plotted the bending response of a microcantilever (with a carboxylate-terminated n-alkanethiol monolayer) to concentration of DIMP molecules corresponding to $63 \mathrm{ppb}$. During the exposure time, we observed no measurable change in the resonance frequency of the microcantilever.

In our present studies, we also used gold-coated microcantilevers to investigate the effect of adsorption of DIMP (on the photo-induced bending of microcantilevers. Depending on the photon wavelength and microcantilever material, the bending can be in either direction. The beauty of this approach is that once analytes have been adsorbed on the surface, the bending can be measured either in real-time, or repeated sometime tater without loss of sensitivity. We measured the photo-induced bending of a

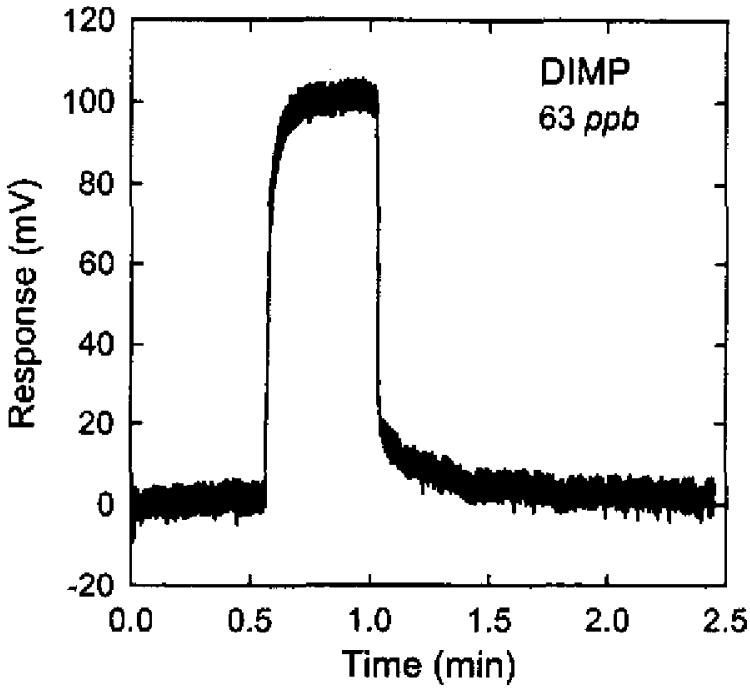

Figure 8. Bending response of a microcantilever with a carboxylate-terminated n-alkanethiol monolayer exposed to DIMP. During the exposure time no measurable change in the resonance frequency was observed.

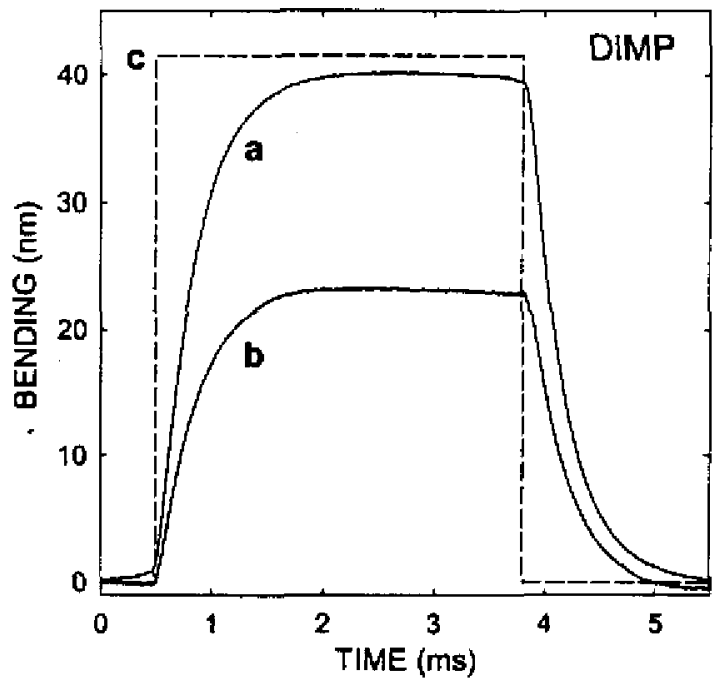

Figure 9. Photo-induced bending of (a) a gold coated microcantilever with no analytes adsorbed, and (b) same microcantilever as before but exposed to DIMP for $60 \mathrm{~s}$. The dashed curve (c) shows the amount of time the microcantilever was exposed to photons that caused the photo-induced bending observed. During the exposure time no measurable change in the resonance frequency was observed. 
gold-coated microcantilever exposed to photons from a diode laser with wavelength $790 \mathrm{~nm}$ [curve (a) in Figure 9]. We subsequently exposed the gold-coated microcantilever to DIMP vapor at room temperature for $60 \mathrm{~s}$. The microcantilevers were exposed to DIMP vapor by placing them into a small vial containing liquid DIMP which at $25^{\circ} \mathrm{C}$ has a vapor pressure of $\sim 700 \mathrm{mTorr}$. Following the adsorption of DIMP molecules, the microcantilever was irradiated using a diode laser ( $790 \mathrm{~nm}$, and same intensity as before) and the measured photo-induced bending is shown by curve (b) in Figure 9. During exposure of the microcantilever to DIMP we did not detect any changes in the resonance frequency $(<0.1 \mathrm{~Hz})$ of the microcantilever placing an upper limit on the adsorbed mass of less that $1 \times 10^{-9} \mathrm{~g} / \mathrm{cm}^{2}$.

\subsection{Trinitrotoluene}

In our studies, we exposed gold-coated Si microcantilevers to TNT molecules in order to investigate the effect of photo-induced stress. The detection of TNT under ambient conditions presents a challenge since TNT is solid at room temperature and has very low vapor pressure $\left(10^{-6} \mathrm{Torr}\right)$. Because of the very low vapor pressure adsorption-induced stress will be very small since only a small number of molecules will be adsorbed on the surface. A small TNT sample (less than $<5 \mathrm{mg}$ ) was placed in a chamber containing a gold-coated microcantilever allowing TNT molecules to adsorbon the microcantilever surface. The TNT sample was removed from the chamber before any measurements were taken. In fact, we found no measurable bending response due to any adsorption-induced stresses from TNT molecules. We measured the photo-induced stress both before and after exposure to TNT using a HeNe laser capable of delivering $10 \mathrm{~mW}$. In Figure 10 we plotted the photoinduced response of the microcantilever prior to exposure to TNT (curve a) and after exposure to TNT (curve b). Figure 10 shows that the photo-induced bending depends on the presence of TNT molecules on the surface. Since Si contracts when exposed to 633 $\mathrm{nm}$ photons, the photo-induced bending decreases as more TNT molecules adsorb on the surface of our detector. We attribute our findings to changes effected in the surface states of the gold-coated microcantilever due to adsorption of TNT molecules.

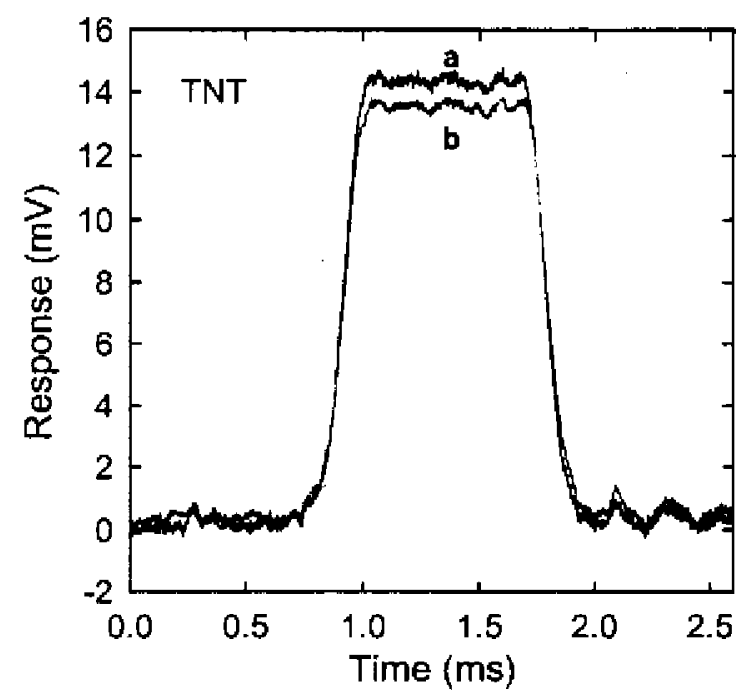

Figure 10. Photo-induced bending of a gold-coated microcantilever to TNT vapor for the same input photon power for (a) no exposure to TNT molecules and (b) exposure to TNT molecules.

In order to estimate the number of TNT molecules adsorbed

on the gold-coated microcantilever surface, we measured the resonance frequency of the microcantilever before it was exposed to TNT molecules. Our results show that initially the resonance frequency was $f_{0}=41.21 \mathrm{kHz}$. However, after TNT molecules adsorbed on the microcantilever surface, we observed no measurable resonance frequency shift. We estimated that the resonance frequency shift would be $<0.01 \mathrm{~Hz}$, placing an upper limit on the adsorbed mass of TNT of less than $10^{16} \mathrm{~g}$ or fewer than $3 \times 10^{7}$ of adsorbed molecules.

\section{CONCLUSIONS}

In this work, we have demonstrated that MEMS based microsystems can be used as sensitive chemical sensors. There are a number of physical and chemical effects that become increasingly important as the physical size of the detector decreases. Monitoring the bending of microcantilevers exposed to target chemicals provides an attractive method for chemical detection. A]though simple chemical coatings can be found, such as gold-coated microcantilevers that respond sensitively to the presence thiols, the selectivity of gravimetric-based detection is a concem. Compounds with small vapor pressure are difficult to detect with simple gravimetric chemical sensors. However, both the selectivity and sensitivity can be tailored using the photo-induced stress in semiconductors and we believe that this limit can be extended to below parts per trillion (ppr). Microcantilevers represent an important development in micromechanical-based chemical detection technology. For example, vastly improved MEMS chemical detectors could be produced by making relatively simple changes in the materials and geometries used in 
microcantilever fabrication. In addition, the compatibility of microcantilever technology with a variety of readout methods affords tremendous flexibility to potential system designers.

\section{ACKNOWLEDGEMENTS}

This work was supported by the DOE EMSP program. Oak Ridge National Laboratory is operated for the U.S. Department of Energy by Lockheed Martin Energy Research Corporation under contract DE-AC05-960R22464.

\section{REFERENCES}

1 D. Sarid, Scanning Force Microscopy with Applications to Electric, Magnetic, and Atomic Forces (Oxford University Press, New York, 1991).

2 J. H. Hoh, J. P. Cleveland, C. B. Prater, J.-P. Revel, and P. K. Hansma, "Quantized Adhesion Detected with Atomic Force Microscope," J. Am. Chem. Soc. 114, 4917 (1992).

3 F. Ohnesorge and G. Binnig, "True Atomic Resolution by Atomic Force Microscopy Through Repulsive Attractive Force," Science 260, 1451 (1993).

4 J. Varesi, J. Lai, T. Perazzo, Z. Shi, and A. Majumdar, "Photothermal Measurements at PicoWatt Resolution Using Uncooled Micro-Optomechanical Sensors," Appl. Phys. Lett. 71, 306 (1997).

5 J. G. E. Harris, D. D. Awschalom, K. D. Maranowski, and A. C. Gossard, "Fabrication and Characterization of 100-nm Thick GaAs Cantilevers," Review of Scientific Instruments 67, 3591 (1996).

6 P. G. Datskos, S. Rajic, I. Datskou, and C. E. Egert, "Infrared MicroCalorimetric Spectroscopy Using Uncooled Thermai Detectors," Infrared Imaging III, SPIE 31 18, 280 (1997).

7 P. G. Datskos, S. Rajic, C. M. Egert, and I. Datskou, in Novel Micro-Calorimetric Spectroscopy Technique for Mine Detection, Monterey, Califomia, Apr. 6-9, 1998.

8 E. A. Wachter and T. Thundat, "Micromechanical Sensors for Chemical and Physical Sensing," Rev, Sci. Instrum 66, 3662 (1995).

9 T. Thundat, G. Y. Chen, R. J. Warmack, D. P. Allison, and E. A. Wachter, "Vapor Detection Using Resonating Microcantilevers." Anal. Chem. 67, 519 (1995).

10 T. Thundat, P. I. Oden, P. G. Datskos, G. Y. Chen, and R. J. Warmack, in Microcantilever Sensors, Oak Ridge Tennessee, 1996.

11 T. Thundat, E. A. Wachter, S. L. Sharp, and R. J. Warmack, "Detection of Mercury Using Resonating Microcantilevers," Appl. Phys. Lett. 66 (1995).

12 P. G. Datskos, S. Rajic, and I. Datskou, "Photo-Induced and Thermal Stress in Si Microcantilevers," Appl. Phys. Lett. 73, 2319 (1998).

13 F. J. von Preissig, "Applicability of the Classical Curvature-stress Relation for Thin Films on Plate Substrates," J. Appl. Phys. 66, 4262 (1989).

14 S. Tomishenko, Theory of Plates and Shells (McGraw-Hill, 1940).

15 G. G. Stoney, "The Tension of Metalic Films deposited by Electrolysis," Proc. Royal Soc. London A 82, 172 (1909).

16 J. Janata, Principles of Chemical Sensors (Plenum, New York, 1989).

17 J. Janata, M. Josowicz, and D. M. Devaney, "Chemical Sensors," Anal. Chem 66, R207 (1994).

18 G. Y. Chen, T. Thundat, E. A. Wachter, and R. J. Warmack, "Adsorption-induced Surface Stress and its Effects on Resonance Frequency of Microcantilevers," J. Appl. Phys. 77, 3618 (1995).

19 P. G. Datskos and I. Sauers, "2-Mercaptoethanol Vapor Sensing with Gold Coated Microcantilevers," Sensors and Actuators B (submitted 1998).

20 L. J. Kepley and R. M. Crooks, "Selective Surface Acoustic Wave-Based Organophosphonate Chemical Sensor Employing a Self-Assembled Composite Monolayer: A paradigm for Sensor Design," Anal. Chem. 64, 3191 (1992). 